\title{
The Effect of Exercise on Bone Mineral Density in Patients with Down Syndrome
}

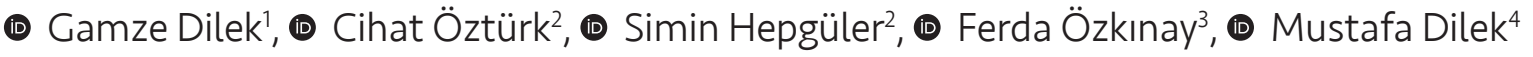 \\ 'Abant İzzet Baysal University, İzzet Baysal Physical Medicine and Rehabilitation Training and Research Hospital, Clinic of Physical Medicine and \\ Rehabilitation, Bolu, Turkey \\ ${ }^{2}$ Ege University Faculty of Medicine, Department of Physical Medicine and Rehabilitation, İzmir, Turkey \\ ${ }^{3}$ Ege University Faculty of Medicine, Department of Pediatrics, Izmir, Turkey \\ ${ }^{4}$ Abant İzet Baysal University Faculty of Medicine, Department of Pediatrics, Bolu, Turkey
}

\begin{abstract}
Aim: Down syndrome (DS) is a predisposing factor for osteoporosis. The aim of this study is to investigate the effect of resistance and jumping exercises on the bone mineral density (BMD) and bone mineral content (BMC) of patients with DS.

Materials and Methods: DS and normal individuals aged between 10 and 30 years old were randomized into an exercise group and a control group: a DS without-exercise group ( $n=15)$, DS exercise group ( $n=17)$, control without-exercise group $(n=18)$, and control exercise group $(n=20)$ were designated. A supervised 45-minute period of exercise was given to the DS exercise group and control exercise group three times per week for six months. The lumbar total and femoral neck BMD and BMC were measured both before and after six months of exercise using dual-energy X-ray absorptiometry. Results: The change in BMD and BMC over six months did not differ significantly between the exercise and control groups. The BMD and BMC of the DS exercise group decreased in the vertebral and femur areas over six months. The study on resistance and jumping exercises over six months did not lead to a significant change in the total BMD and BMC in the vertebral and femoral neck areas of the patients with DS.

Conclusion: The decrease in BMD and BMC over six months in the DS exercise group is not a predictable result.

Keywords: Down syndrome, exercise on bone mineral density, childhood, adult
\end{abstract}

\section{Introduction}

Physical activity is important for bone formation in order to maintain bone mass. Exercise training has positive effects on peak bone mass during childhood. An active life style and high-intensity physical activity are recommended to reduce the risk of osteoporosis in adulthood (1). Down syndrome (DS) is one of the most common types of genetic abnormalities related to disability. Hypotonia and joint hyper-flexibility are skeletal problems that cause morbidity in people with DS (2). Osteoporosis risk increases in people with DS as they age (3). However, the life expectancy of people with DS has increased recently due to improvements in health care. Mechanotransduction is bone formation due to mechanical stress. Mechanical stimuli cause the stimulation of osteocyte proliferation and the differentiation of osteoblasts, collagen synthesis and bone mineralization (4). For these reasons, we preferred resistance training and intensive physical activities as the exercise type for our study. Resistance training is effective in maintaining and increasing bone mineral density (BMD) (5). Intensive physical activities, such as basketball, jumping, and gymnastics, are effective in increasing vertebral

\section{Address for Correspondence}

Gamze Dilek MD, Abant Izzet Baysal University, Izzet Baysal Physical Medicine and Rehabilitation Training and Research Hospital, Clinic of Physical Medicine and Rehabilitation, Bolu, Turkey Phone: +90 5054471786 E-mail: gamzedilekk@gmail.com ORCID ID: orcid.org/0000-0002-0029-9933

Received: 05.06 .2017 Accepted: 19.03 .2018

${ }^{(\odot)}$ Copyright 2018 by Ege University Faculty of Medicine, Department of Pediatrics and Ege Children's Foundation The Journal of Pediatric Research, published by Galenos Publishing House. 
and hip BMD while physical activity is important for gaining and providing bone mass $(6,7)$. Children with DS have low BMD. Hypotonia and decreasing muscle strength in DS may lead to decreased bone mass. The purpose of this study is to assess the effect of loading exercise treatment on the BMD of patients with DS.

\section{Materials and Methods}

\section{Design}

The initial study population consisted of 40 male and 40 female subjects (10-30 years old); 40 with DS; and 40 healthy controls. The mildly mental retarded DS participants, were recruited from two special schools by advertisement, and the healthy group attended high schools and universities in İmir. The treatment group was divided into an exercise group and a control group. The control group consisted of 20 DS (mean age $19.80 \pm 4.1$ years) and 20 healthy people (mean age 20.0 \pm 4.9 ). The exercise group consisted of 20 DS with a mean age of $22.01 \pm 2$ years and 20 healthy people with a mean age of $20.0 \pm 4.2$ years. The study was completed with 70 subjects in six months [two subjects in the healthy control group and five subjects in the DS control group did not accept the second dual-energy X-ray absorptiometry (DXA) measurement; three subjects in the DS exercise group could not complete the exercise study]. DXA measurements were performed to evaluate the benefit of the exercise treatment after six months of training. The third DXA measurement could not be performed in seven subjects in the healthy control group and eight subjects in the healthy exercise group due to not being available at the given time. The study was completed with 55 subjects within 12 months. Patients in the DS group were living with their families under good dietary and environmental conditions. The individuals in all groups were euthyroid. The DS, healthy men and women neither had systemic diseases nor were on any medication, vitamin or mineral affecting bone mineralization. The blood calcium and 25-hydroxyvitamin D (25 OHD) levels of participants were not evaluated before the study. Musculoskeletal deformities were not ascertained. All participants were non-smokers and they did not use alcohol during the study period. Informed consent was obtained from all subjects and their parents. The study was approved by the Ege University Local Ethics Committee (approval number: 08-12.1/14).

\section{Participants, Therapists}

The loading and resistive exercise training program consisted of three 45-minute exercise sessions per week for six months. The training included the following exercises: back extension exercise, squatting, resistance hip abduction, trunk flexion, bridge, side bridge, abdominal curl-up, jumping and upper extremity resistive exercise. The lifting weight was determined as $50-70 \%$ of their one repetition maximum and was not increased during the study. Jumping exercises were repeated 100 times per session. The resistive training was repeated 10 times for each exercise type. The exercise treatment was given to the healthy training group as a home program that was followed up on via phone call from the supervisor physician. The exercise group with DS was trained by the supervisor physician during the first three months and was trained by a physical education teacher while simultaneously being monitored by the supervisor physician during the second three months. The exercise treatment for subjects with DS was given in their schools.

\section{Outcome Measures}

The bone mineral content (BMC) g, BMD gr/ $\mathrm{cm}^{2}$ and bone area $\mathrm{cm}^{2}$ were determined using DXA (HOLOGIC 4500 A). The BMD, BMC and bone area were measured at the beginning of the study, at six and twelve months after the start. BMD, BMC and bone area of the lumbar spine (L1-4, anteroposterior), femoral neck, and femur total were derived using spine software and femur software. In our laboratory, the intra-class correlations for repeated measurements, including the subregions, were 0.933 for femoral neck BMD, 0.975 for femoral neck BMC, 0.880 for femur total BMD, 0.985 for femur total BMC, 0.990 for lumbar total BMD and 0.996 for lumbar total BMC.

\section{Statistical Analysis}

The comparisons between the patients and controls were made using the t-test or the Mann-Whitney $U$ test in the case of significant deviation from the normal distribution. A one-way analysis of variance (ANOVA) was used to test the hypotheses regarding the equality means between the groups for the following subject characteristics: age, body weight, height and body mass index (BMI). The Bonferroni post-hoc test was used to determine the differences between the groups. Intention to treat analysis was used for missing data at the first year of the study, with the sixth month's data of the missing subjects used for the analysis.

\section{Results}

The characteristics of the subjects, such as weight, age, and $\mathrm{BMI}$, were similar at the baseline and at the completion of the study. The height of the DS control group was significantly lower than the two healthy groups (Table I). The baseline lumbar spine BMD and BMC as well as the femoral neck and total BMD and BMC of the DS group were significantly lower than those of the healthy groups (Table II). During the study period, BMD and BMC values of the lumbar spine and femoral neck showed no significant difference between patient and control groups, and no significant difference was observed in any of these three locations between the initial and posttraining values (Table II). The BMD values in the lumbar spine, femoral neck and total femur decreased in the DS exercise 
Table I. Demographic characteristics of the study groups

\begin{tabular}{|l|l|l|l|l|}
\hline & Normal control & Normal exercise & DS control & DS exercise \\
\cline { 2 - 5 } & $\mathbf{( n = 1 8 )}$ & $\mathbf{( n = 2 0 )}$ & $\mathbf{( n = 1 5 )}$ & $\mathbf{( n = 1 7 )}$ \\
\hline Age & $20.11 \pm 5.21$ & $19.65 \pm 4.49$ & $19.60 \pm 5.93$ & $22.41 \pm 2.69$ \\
\hline Initial weight & $63 \pm 14.49$ & $59.5 \pm 18.94$ & $58.74 \pm 18.64$ & $64.2 \pm 13.4$ \\
\hline Weight at 6 ${ }^{\text {th }}$ month & $64.4 \pm 14.5$ & $60.6 \pm 17.5$ & $58.6 \pm 16.5$ & $64.54 \pm 13.5$ \\
\hline Weight at first year & $65.4 \pm 14.2$ & $58.9 \pm 15.3$ & $59 \pm 17$ & $64.7 \pm 12$ \\
\hline Initial height & $165.8 \pm 2.7$ & $165.6 \pm 2.0$ & $151.2 \pm 2.1^{*}$ & $158.4 \pm 1.4$ \\
\hline Height at 6 ${ }^{\text {th }}$ month & $166.4 \pm 2.6$ & $165.9 \pm 1.9$ & $151.5 \pm 2.0^{*}$ & $159 \pm 1.2$ \\
\hline Height at first year & $168.7 \pm 2$ & $164.4 \pm 2$ & $151.1 \pm 1^{*}$ & $158.5 \pm 2$ \\
\hline Initial BMl & $22.6 \pm 0.74$ & $21.4 \pm 1.1$ & $25.1 \pm 1.7$ & $25.4 \pm 1.0$ \\
\hline BMl at 6 ${ }^{\text {th }}$ months & $23.0 \pm 0.80$ & $21.7 \pm 0.9$ & $21.4 \pm 0.6$ & $25.4 \pm 1.0$ \\
\hline BMl at first year & $23.4 \pm 1$ & $25.6 \pm 1.4$ & $25.5 \pm 0.9$ \\
\hline
\end{tabular}

BMD: Bone mineral density, BMC: Bone mineral content, BMI: Body mass index, DS: Down syndrome ${ }^{*} \mathrm{p}<0.05$

group at the sixth month of the study; despite this, these values were increased non-significantly at the sixth month in the other three groups. The lumbar spine and femoral neck $B M C$ values of the DS exercise group decreased at the sixth month of the study, while the BMC values of the other three groups increased (Table II). The changes in the BMC values were not significant. The BMD and BMC values of the DS exercise group, which decreased at the sixth month, showed a non-significant increase at the end of the first year of the study.

\section{Discussion}

Several studies have determined that individuals with DS have reduced BMD in comparison to healthy controls $(8,9)$. Several investigators determined that adynamic bone formation takes place in people with DS (10). The present study shows that people with DS have lower BMD and BMC in the spine and hip than to normal controls, which is in accord with the results of previous studies. In the study of Angelopoulou et al. (11), the lumbar BMD in men and women with DS were $25 \%$ lower than those of normal controls; Baptista et al. (12) reported that subjects with DS had 20\% lower lumbar BMC than the healthy subjects. In our study, the lumbar BMC, femoral neck and femur total were lower in patients with DS compared with normal controls. Angelopoulou et al. (11) observed that the muscle strength of mentally retarded people with DS was lower than that of healthy people and mentally retarded people without DS. Matute-Llorente et al. (13) determined that adolescents with DS who perform longer periods of physical activity have higher BMD Z-scores than those who perform less physical activity. Experimental studies indicate that high-impact activities should lead to high mechanic stimuli and thus be over the osteogenic index (14). It is reported that the jumping exercises are good for femur and vertebra BMD (14). In the study of Fuchs et al. (15), the femoral and vertebral BMD of healthy subjects were higher than those of the control group, who jumped over the $61 \mathrm{~cm}$ sized box; the ground reaction force was determined to be 8.8 more than their body weights. Witzke et al. (16) observed that the femoral neck and trochanter BMD were higher in the exercise group than in the controls after a plyometrics jumping exercise study that took place over nine months, with exercises being completed three times a week and 360 times per exercise session. The present study involved jumping exercises 100 times per exercise session, three times a week. The ground reaction force was determined to be 3-4 times more than the subjects' body weights with the jumping style used in our present study as in previous studies. Experimental studies indicate that the load on an L4-5 vertebra is about $2200 \mathrm{~N}$ during the two-leg-raising and trunk flexion positions. At the same time, the load on the femur and lumbar vertebra is six times more than the body weight during the squat exercise (17). Ferry et al. (18) determined a significant increase at the lumbar spine, total hip BMC and lumbar spine BMD after a one-year physical training program study. Nichols et al. (19) observed an increase in BMD at the femoral neck but not with the lumbar total of adolescent girls during a 15 month resistance exercise program. In the study of González-Agüero et al. (20), which consisted of 25 minute sessions per week 


\begin{tabular}{|c|c|c|c|c|c|}
\hline & & Normal control & Normal exercise & DS control & DS exercise \\
\hline & & $n=18$ & $\mathrm{n}=\mathbf{2 0}$ & $n=15$ & $n=17$ \\
\hline \multirow[t]{3}{*}{ Lumbar total BMD } & Initial & $0.936 \pm 0.165$ & $0.916 \pm 0.14$ & $0.795 \pm 0.149$ & $0.871 \pm 0.136$ \\
\hline & $6^{\text {th }}$ month & $0.964 \pm 0.156$ & $0.942 \pm 0.129$ & $0.815 \pm 0.136$ & $0.868 \pm 0.149$ \\
\hline & First year & $0.960 \pm 0.150$ & $0.950 \pm 0.150$ & $0.830 \pm 0.120$ & $0.870 \pm 0.130$ \\
\hline \multirow[t]{3}{*}{ Femur neck BMD } & Initial & $0.827 \pm 0.142$ & $0.825 \pm 0.114$ & $0.794 \pm 0.124$ & $0.827 \pm 0.129$ \\
\hline & $6^{\text {th }}$ month & $0.844 \pm 0.152$ & $0.838 \pm 0.135$ & $0.799 \pm 0.15$ & $0.814 \pm 0.129$ \\
\hline & First year & $0.850 \pm 0.130$ & $0.840 \pm 0.120$ & $0.810 \pm 0.100$ & $0.820 \pm 0.120$ \\
\hline \multirow[t]{3}{*}{ Femur total BMD } & Initial & $0.942 \pm-0.182$ & $0.921 \pm 0.121$ & $0.844 \pm 0.133$ & $0.877 \pm 0.129$ \\
\hline & $6^{\text {th }}$ month & $0.956 \pm 0.156$ & $0.935 \pm 0.133$ & $0.862 \pm 0.169$ & $0.876 \pm 0.136$ \\
\hline & First year & $0.968 \pm 0.156$ & $0.930 \pm 0.120$ & $0.870 \pm 0.169$ & $0.880 \pm 0.136$ \\
\hline \multirow[t]{3}{*}{ Lumbar total BMC } & Initial & $57.96 \pm 19.38$ & $54.38 \pm 15.00$ & $42.66 \pm 12.52$ & $50.2 \pm 12.93$ \\
\hline & $6^{\text {th }}$ month & $59.84 \pm 18.07$ & $56.21 \pm 15.27$ & $44.77 \pm 12.27$ & $49.89 \pm 11.99$ \\
\hline & First year & $60.20 \pm 17.04$ & $57.33 \pm 15.33$ & $46.15 \pm 15.27$ & $50.80 \pm 12.03$ \\
\hline \multirow[t]{3}{*}{ Femur neck BMC } & Initial & $4.33 \pm 1.05$ & $4.21 \pm 13.27$ & $3.54 \pm 0.66$ & $3.78 \pm 0.89$ \\
\hline & $6^{\text {th }}$ month & $4.49 \pm 1.10$ & $4.46 \pm 0.98$ & $3.71 \pm 0.77$ & $3.68 \pm 0.92$ \\
\hline & First year & $4.49 \pm 1.10$ & $4.49 \pm 0.94$ & $3.79 \pm 0.72$ & $3.76 \pm 0.72$ \\
\hline \multirow[t]{3}{*}{ Femur total BMC } & Initial & $3.36 \pm 11.72$ & $32.12 \pm 9.06$ & $25.44 \pm 8.01$ & $28.14 \pm 7.46$ \\
\hline & $6^{\text {th }}$ month & $33.72 \pm 11.43$ & $33.57 \pm 9.88$ & $26.26 \pm 7.86$ & $28.58 \pm 7.83$ \\
\hline & First year & $27.33 \pm 17.03$ & $31.69 \pm 10.08$ & $26.78 \pm 7.00$ & $28.48 \pm 7.48$ \\
\hline Mean \pm SD & - & - & - & - & - \\
\hline
\end{tabular}

BMD: Bone mineral density, BMC: Bone mineral content, SD: Standard deviation, DS: Down syndrome

of a conditioning and plyometric jump training program for 21 weeks, increases in the total and hip-region BMC in the DS exercise group were found compared with the DS non-exercise group in the training program. In this study, we considered the previous exercise studies on bone formation and decided to perform the jumping and resistance exercise experimentation for patients with DS in order to increase their BMD and BMC. However, the results of the present study did not comply with the previous studies. We did not find a significant increase in the lumbar spine and femoral BMD and BMC in the exercise group. Chilibeck et al. (21) did not observe an increase in BMD at the lumbar spine and in the femur of young adults during a 20-week resistance exercise study. It was reported that the osteogenic effect on bone is greater when implementing the jumping and resistance exercises together (14). We performed both jumping and resistance training for two groups. Soomro et al. (22) did not observed any significant difference on young females who were given osteoporosis preventing exercises versus walking. They have postulated that the short duration of the study and small sample size resulted in a non-significant outcome. In the present study, we observed a non-significant increase in the lumbar total, femoral neck and total BMD and $B M C$ in the healthy study group, healthy control group and control group with DS, but we observed a non-significant decrease in the lumbar total and femoral neck BMD and BMC in the study group with DS at the sixth month of the study. Matute-Lorente et al. (23) observed non-significant increase in $B M C$ and BMD values with DS while healthy controls showed more significant changes after undergoing whole body vibration training. They suggested that exercise training might have higher bone resorption and lower bone formation. Puustjärvi et al. (24) demonstrated that a reorganization in bone collagen with mechanic loading increases bone strength; however, they reported that a BMD decrease does not affect bone strength after mechanic loading (25). No study has researched the collagen structure of DS patients and the relationship between collagen structure and BMD. McKelvey et al. (26) determined that adults with DS had lower bone 
formation markers than healthy adults. They claimed that these markers are significantly associated with low BMD in people with DS. It was supposed that the insufficient trabecular connection in bone in people with DS is due to a decreased message transmission between bone cells, which causes a mineralization defect (25). In the present study, the non-dynamic bone structure and lower bone formation of the subjects with DS might have caused an insufficient response of the bone to mechanic loading. The femoral neck, which contains less trabecular bone than a vertebra, might be insufficient with respect to the micro damage during the jumping exercise sessions. The exercise protocol was taught to the healthy exercise group as a home program. The healthy exercise group was controlled by the researchers via phone call on a weekly basis which decreased the study value. Another limitation of our study is the patient number. We could not reach the patient number that was needed. In our study, we observed a non-significant increase in all the parameters of the healthy groups and DS control group, while there was a non-significant decrease in the BMD and BMC of the DS exercise group. In the present study, we could not analyze the bone formation markers, blood calcium and $25 \mathrm{OHD}$.

\section{Conclusion}

Individuals with DS have an increased risk of osteoporosis. Weight bearing exercises might be designed and applied considering their special musculoskeletal structure in order to prevent osteoporosis. Despite the limitations of this study, the present study can be useful in the making of specific exercise protocols for patients with DS. More detailed and longer duration studies that contain more subjects are needed to express the frequency and intensity of exercise required for people with DS who are at risk for osteoporosis and low bone density.

\section{Acknowledgements}

The authors thank: All participants' parents and all participants; İzmir National Education Directorate; Manisa National Education Directorate. No financial assistance was provided for this study.

\section{Ethics}

Ethics Committee Approval: The study was approved by the Ege University Local Ethics Committee (approval number: 08-12.1/14).

Informed Consent: Consent form was filled out by all participants.

Peer-review: External and internal peer-reviewed.

\section{Authorship Contributions}

Surgical and Medical Practices: G.D., C.Ö., Concept: G.D., C.Ö., Design: G.D., C.Ö., F.Ö., Data Collection or Processing: G.D., S.H., Analysis or Interpretation: G.D., M.D.,
C.Ö., S.H., Literature Search: G.D., M.D., Writing: G.D., M.D., C.Ö., S.H.

Conflict of Interest: No conflict of interest was declared by the authors.

Financial Disclosure: The authors declared that this study received no financial support.

\section{References}

1. Vlachopoulos D, Barker AR, Williams CA, et al. Effect of a program of short bouts of exercise on bone health in adolescents involved in different sports: the PRO-BONE study protocol. BMC public health 2015;15:361.

2. Hayes A, Batshaw ML. Down syndrome. Pediatric clinics of North America 1993;40:523-35.

3. Kao $\mathrm{CH}$, Chen CC, Wang SJ, Yeh SH. Bone mineral density in children with Down's syndrome detected by dual photon absorptiometry. Send to Nucl Med Commun 1992;13:773-5.

4. Frost HM. Bone's mechanostat: a 2003 update. Anat Rec A Discov Mol Cell Evol Biol 2003;275:1081-101.

5. Kelley GA, Kelley KS, Tran ZV. Resistance training and bone mineral density in women: a meta-analysis of controlled trials. Am J Phys Med Rehabil 2001;80:65-77.

6. Burrows M. Exercise and bone mineral accrual in children and adolescents. I Sports Sci Med 2007;6:305-12.

7. Heinrich $\mathrm{CH}$, Going SB, Pamenter RW, et al. Bone mineral content of cyclically menstruating female resistance and endurance trained athletes. Med Sci Sports Exerc 1990;22:558-63.

8. Angelopoulou N, Souftas V, Sakadamis A, Mandroukas K. Bone mineral density in adults with Down's syndrome. Eur Radiol 1999;9:648-51.

9. Guijarro M, Valero C, Paule B, Gonzalez-Macias I, Riancho JA. Bone mass in young adults with Down syndrome. I Intellect Disabil Res 2008;52:182-9.

10. Tumer Z, Henriksen AM, Bache I, et al. Eponymous Jacobsen syndrome: mapping the breakpoints of the original family suggests an association between the distal $1.1 \mathrm{Mb}$ of chromosome 21 and osteoporosis in Down syndrome. Am J Med Genet A 2005;135:339-41.

11. Angelopoulou N, Matziari C, Tsimaras V, et al. Bone mineral density and muscle strength in young men with mental retardation (with and without Down syndrome). Calcif Tissue Int 2000;66:176-80.

12. Baptista F, Varela A, Sardinha LB. Bone mineral mass in males and females with and without Down syndrome. Osteoporos Int 2005; 16:380-8.

13. Matute-Llorente A, González-Agüero A, Gómez-Cabello A, Vicente-Rodríguez G, Casajús JA. Decreased levels of physical activity in adolescents with down syndrome are related with low bone mineral density: a cross-sectional study. BMC Endocr Disord 2013;13:22.

14. Stengel SV, Kemmler W, Pintag R, et al. Power training is more effective than strength training for maintaining bone mineral density in postmenopausal women. / Appl Physiol 2005;99:181-8.

15. Fuchs RK, Bauer IJ, Snow CM. Jumping improves hip and lumbar spine bone mass in prepubescent children: a randomized controlled trial. J Bone Miner Res 2001;16:148-56.

16. Witzke KA, Snow CM. Effects of plyometric jump training on bone mass in adolescent girls. Med Sci Sports Exerc 2000;32:1051-7. 
17. Granata KP, Lee PE, Franklin TC. Co-contraction recruitment and spinal load during isometric trunk flexion and extension. Clin Biomech 2005;20:1029-37.

18. Ferry B, Gavris M, Tifrea C, et al. The bone tissue of children and adolescents with Down syndrome is sensitive to mechanical stress in certain skeletal locations: a 1-year physical training program study. Res Dev Disabil 2014;35:2077-84.

19. Nichols DL, Sanborn CF, Love AM. Resistance training and bone mineral density in adolescent females. J Pediatr 2001;139:494500.

20. González-Agüero A, Vicente-Rodríguez G, Gómez-Cabello A, et al. A 21-week bone deposition promoting exercise programme increases bone mass in young people with Down syndrome. Dev Med Child Neurol 2012;54:552-6.

21. Chilibeck PD, Paterson DH, Smith WD, Cunningham DA. Cardiorespiratory kinetics during exercise of different muscle groups and mass in old and young. I Appl Physiol 1996;81:1388-94.
22. Soomro RR, Ahmed SI, Khan M, Ali SS. Comparing the effects of Osteoporosis Prevention Exercise Protocol (OPEP) versus walking in the prevention of osteoporosis in younger females. Pak J Med Sci 2015;31:336-40.

23. Matute-Llorente A, González-Agüero A, Gómez-Cabello A, et al. Effect of whole-body vibration training on bone mass in adolescents with and without Down syndrome: a randomized controlled trial. Osteoporos Int 2016;27:181-91.

24. Puustjärvi K, Nieminen ), Räsänen $T$, et al. Do more highly organized collagen fibrils increase bone mechanical strength in loss of mineral density after one-year running training? I Bone Miner Res 1999;14:321-9.

25. Grimwood JS, Kumar A, Bickerstaff DR, Suvarna SK. Histological assessment of vertebral bone in a Down's syndrome adult with osteoporosis. Histopathology 2000;36:279-80.

26. McKelvey KD, Fowler TW, Akel NS, et al. Low bone turnover and low bone density in a cohort of adults with Down syndrome. Osteoporos Int 2013;24:1333-8. 